\title{
Understanding the Impact of Maternal HIV Infection on the Health and Well-Being of Mothers and Infants in South Africa: Siyakhula Collaborative Workshop Report
}

\author{
Marina White' \\ Ute D Feucht $\mathbb{D}^{2-4}$ \\ Louise de Villiers du Toit ${ }^{5}$ \\ Theresa Rossouw $\mathbb{D i D}^{3-5}$ \\ Kristin L Connor' \\ 'Health Sciences, Carleton University, \\ Ottawa, Ontario, Canada; ${ }^{2}$ Paediatrics, \\ University of Pretoria, Pretoria, Gauteng, \\ South Africa; ${ }^{3}$ Research Centre for \\ Maternal, Fetal, Newborn \& Child Health \\ Care Strategies, University of Pretoria, \\ Pretoria, Gauteng, South Africa; \\ ${ }^{4}$ Maternal and Infant Health Care \\ Strategies Unit, South African Medical \\ Council, Pretoria, Gauteng, South Africa; \\ ${ }^{5}$ Department of Immunology, University \\ of Pretoria, Pretoria, Gauteng, South \\ Africa
}

\begin{abstract}
The Siyakhula study is an ongoing, observational cohort study in Pretoria, South Africa, that aims to understand how maternal HIV infection and perinatal environmental factors shape development and health in infants who are HIV-exposed (in utero and during breastfeeding) but remain uninfected themselves (HEU). The Siyakhula Collaborative Workshop, which took place at the Research Centre for Maternal, Fetal, Newborn \& Child Health Care Strategies at Kalafong Hospital in Pretoria, South Africa on November 15-16, 2018, brought together a group of international health scientists, clinicians, and stakeholders, including women with lived experience, to build capacity for research and training on the impact of HIV infection on women's and infants' health across geographical and disciplinary boundaries. The workshop sought to summarise the state of knowledge on the effects of being HEU on infant development and health in the first two years of life, identify gaps in existing research on modifiable exposures that may be associated with poor infant development, and develop ideas for novel research and interventions to lessen or prevent adverse health outcomes in pregnant or breastfeeding people living with HIV. These proceedings summarise the pre-workshop consensus process that was used to identify priority areas to discuss during small-group breakout sessions, as well as the themes and key challenges that emerged from these discussions during the workshop.
\end{abstract}

Keywords: HIV, PMTCT, breastfeeding, growth, neurodevelopment, immune

\section{Introduction}

In recent decades, targeted public health programmes have increased the availability of antiretroviral therapies (ART) to reduce mother-to-child transmission (MTCT) of HIV, reaching around $85 \%$ of pregnant people living with HIV in 2019. ${ }^{1}$ These global efforts to prevent MTCT have improved pregnancy outcomes for women living with HIV, including reductions in the rates of stillbirth, preterm birth and low birthweight. ${ }^{2}$ Further, the number of children who are HIV exposed (in the womb and during breastfeeding), but uninfected (HEU) themselves, is also rising, ${ }^{3}$ reaching an estimated 14.8 million in $2018 .^{4}$ Evidence suggests that children who are HEU experience poorer growth and development ${ }^{5-7}$ compared to children who have not been exposed to maternal HIV infection. However, the mechanisms driving poorer health and developmental outcomes in children who are HEU are not fully understood and, despite improved ART access for pregnant 
and breastfeeding people, challenges with treatment adherence $^{8}$ or concurrent adversities, including malnutrition, ${ }^{9}$ food insecurity ${ }^{10}$ and low socioeconomic status, ${ }^{11}$ may exacerbate poor health outcomes. Thus, a multidisciplinary, integrated approach is required to improve the health and well-being of mothers and infants affected by HIV.

The Siyakhula study is an ongoing, observational cohort study at Kalafong Provincial Tertiary Hospital in Pretoria, South Africa. It aims to understand how maternal HIV infection and ART in combination with environmental factors during pregnancy and postnatally, such as maternal nutrition, breastmilk and breastfeeding practices, and food and nutrition security, shape development and health of infants who are HEU. Siyakhula means to grow, in isiZulu, and represents the growing need to lessen the impact and lasting burden of infant exposure to HIV in utero and during breastfeeding for optimal development and health of future generations. This aligns closely with the developmental origins of health and disease risk and resilience across the lifecourse, ${ }^{12}$ and the urgent need to promote and support a healthy start to life.

The Siyakhula Collaborative Workshop was held around the Siyakhula study's outset on November 15-16, 2018, at the University of Pretoria's Research Centre for Maternal, Fetal, Newborn and Child Health Care Strategies at Kalafong Provincial Tertiary Hospital. The purpose of the workshop was to build the capacity of local and international researchers and community members to participate in, and contribute to, research pertaining to the impact of HIV infection on maternal, newborn, infant and child health. Attendees included study participants, to ensure the development of patient-important research questions and improve the translatability of research finding, ${ }^{13}$ as well as clinicians, health scientists and community services representatives.

The specific aims of the workshop were to:

1. Summarise the state of knowledge on HIV and ART exposure in utero, without infant infection, on infant development and health in the first two years of life;

2. Identify gaps in existing research on modifiable exposures that may be associated with infant growth, neuro- and immunological development, including risk for infectious diseases;

3. Improve our understanding of the health needs of women living with HIV and their infants; and
4. Review current strategies to lessen or prevent adverse health outcomes, including the presence of multiple comorbidities, in women living with HIV and their infants who are HEU, and develop ideas for novel research and interventions.

A series of presentations and small-group breakout discussions took place to achieve these workshop aims. This paper summarises the pre-workshop consensus process that was used to identify priority areas for discussion and the themes, key challenges, and opportunities for strengthening research that emerged from these discussions during the workshop.

\section{Materials and Methods Ethics and Consent}

This workshop report was exempt from ethics approval by the Carleton University Research Ethics Board. All workshop attendees were made aware at the workshop outset that discussions would be synthesized in a report to keep record of the meeting.

\section{Participants}

The 33 individuals in attendance at the workshop were comprised of women who are living with HIV and are participants in the Siyakhula study $(\mathrm{n}=2)$, community health workers (Ward-based outreach teams $[n=1]$ ), representatives from community programmes and services (Tshwane District Health Services Maternal-Child [ $\mathrm{n}=1]$ and Child Health programmes [ $\mathrm{n}=1]$ ) and non-profit or non-governmental organizations $(n=2)$, Siyakhula study research assistants $(n=3)$, and research scientists (from the fields of Immunology $[n=2]$; Virology $[n=1]$; Epidemiology $[\mathrm{n}=2]$; Statistics $[\mathrm{n}=1]$; Developmental Origins of Health and Disease [n=1]; Nutrition and Food Science $[n=2]$ ), physicians (from the disciplines of Pediatrics [n=2]; Obstetrics [n=1]; Maternal-Fetal Medicine [n=1]; Family Medicine [n=1]), nurses $(n=1)$, graduate students $(n=5)$, dieticians $[n=1]$, and representatives from regional and national government institutions $(n=2)$. There were 30 individuals in attendance for both days of the workshop.

\section{Pre-Workshop Survey}

A pre-workshop survey was developed using an adapted Delphi technique. ${ }^{14-16}$ The aim was to provide a structured environment where interdisciplinary stakeholders were 
given an opportunity to share expertise and input on priority topics to be covered during the workshop. ${ }^{14}$ The Delphi technique is used to gather individual, anonymized opinions of group participants over a series of survey rounds, often administered through email. After each round, the results are aggregated and shared with the group, and the process is considered complete when there is convergence of group opinion and consensus is reached. ${ }^{14,17}$

The pre-workshop survey questions were developed in collaboration with researchers and clinicians based in South Africa and Canada (Table 1) and consisted of two rounds of participant input. The first round was distributed to expected workshop attendees through an email invitation via Qualtrics (an online survey administration system; Provo, Utah, USA) approximately one month before the workshop (Figure 1). The responses from the first round were then aggregated, collapsing stakeholder responses into common themes. For example, "Safety of antiretroviral therapy (ART) used during pregnancy", "Information on ART for mothers - why, how, side-effects, adherence", and "Understanding what effect antiretroviral drugs have on maternal and child health outcomes" (responses to question 1, Table 1), were collapsed into "ART education, safety and adherence".

\section{Workshop Survey}

A second round of participant input was gathered using an adapted nominal group technique, which provides a structured, in-person environment where participants individually list ideas related to the given topic being investigated and then share their priority idea or concern with the group. ${ }^{14,16,18}$ Once all ideas are presented, group discussion commences, and participants rank the ideas shared by group members until the priority topics are agreed upon.

At the start of the workshop, the aggregated results from the first survey were presented to the group in-person, highlighting the answers that were currently ranked as the top three responses to each question. Participants were asked to express whether they agreed or disagreed with the rankings, and to identify any topics that they believed were missing. Following these discussions, final priority themes were identified from items with the highest ranking (based on the number of votes received) and set as guiding topics for small-group breakout discussions.

\section{Small-Group Breakout Sessions}

Workshop attendees were randomly divided into three breakout groups composed of 10 participants each and remained in these groups for each of the three small-
Table I Pre-Workshop Survey (Round I) Distributed to Participants via Email

I. Presently, what key concerns must be addressed when aiming to improve the health and well-being of mothers and infants affected by HIV? Please provide 3-6 concerns.

2.What are $\mathbf{3}$ significant key challenges you foresee for the future of maternal-infant HIV research?

3. What $\mathbf{3}$ key health outcomes are important to measure when assessing health trends of infants and mothers affected by HIV?

4. If you were to design an interdisciplinary research programme aimed at improving the health outcomes of mothers and/or infants affected by HIV, what components would be most important to ensure the success of that programme in reaching its goals? These components may incorporate, amongst others: specific professional/disciplinary specialties, or certain research designs that you would include.

5. What pre-conception or pregnancy factors must be evaluated and addressed in order to enhance the pregnancy environment in women affected by HIV? Please rank the following topics (I-9) in terms of importance ("I" being the most important):

- Maternal mental health and well-being

- Maternal physical health (eg HIV status, weight, other disorders/ disease, medication use)

- Home environment

- Support from partner, family, and/or community

- Maternal level of education attained

- Maternal socio-economic characteristics

- Food security and/or quality of food

- Accessibility to antenatal care

- Other [fillable]

6. What postpartum factors must be evaluated and addressed in order to enhance development of infants and children affected by HIV? Please rank the following topics (I-I6) in terms of importance ("I" being the most important):

- Maternal mental health and well-being

- Maternal physical health (eg HIV status, weight, infection, other disorders/disease, medication use)

- Home environment

- Support from partner, family, and/or community

- Maternal level of education attained

- Maternal socio-economic characteristics

- Food security and/or quality of food

- Breastfeeding practices

- Infant language development

- Infant cognitive development

- Infant motor development

- Infant behavioural development

- Infant growth

- Infant susceptibility to infection and disease

- Accessibility of postnatal care

- Other [fillable]

7. What strategies for intervention do you think are essential to prevent or lessen the adverse impacts of HIV-exposure for women living with HIV, and their infants? 


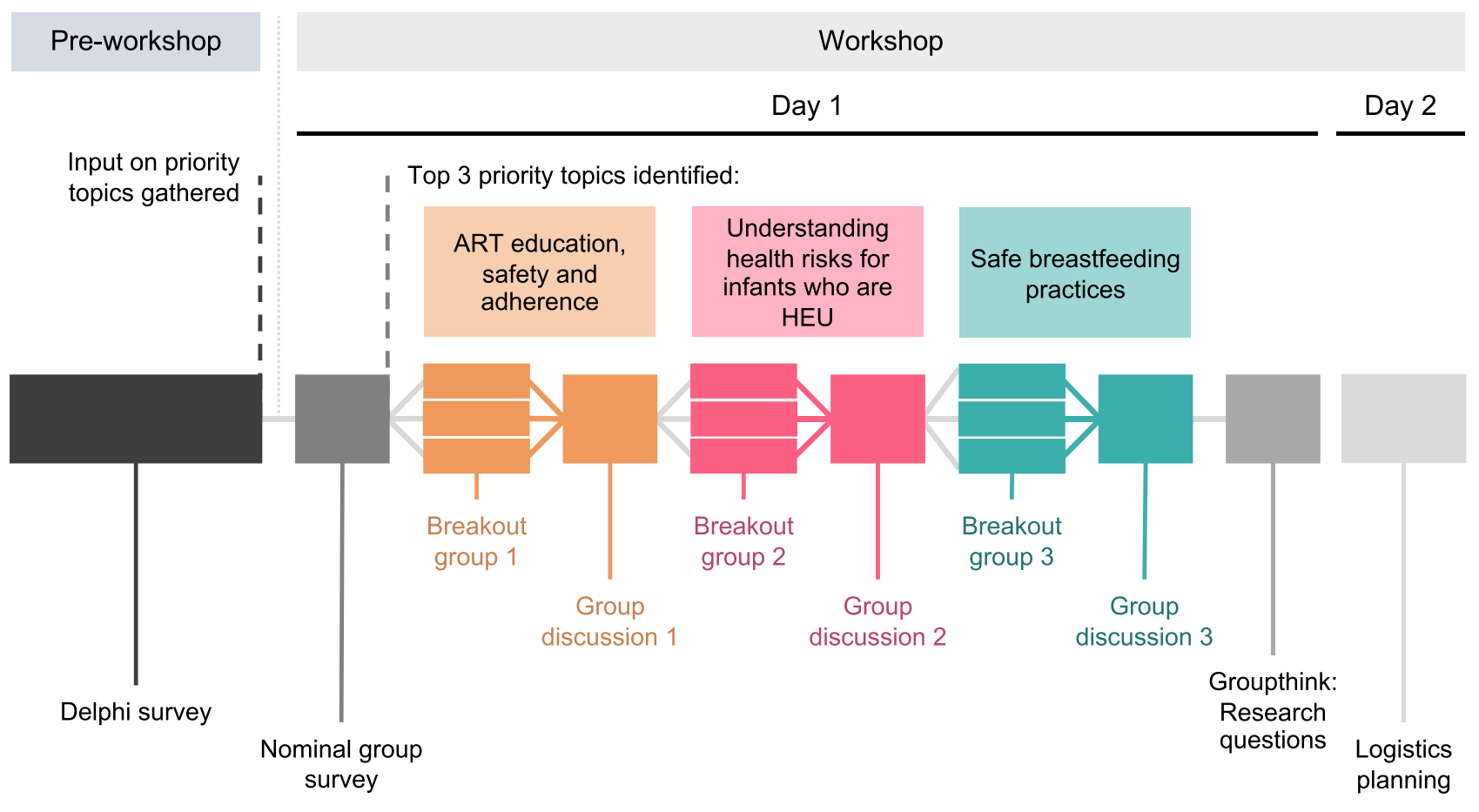

Figure I Timeline of pre-workshop and workshop activities.

group discussion sessions. Each breakout session had a rapporteur who was tasked with recording minutes for the breakout session and sharing discussion highlights upon reconvening with the whole group. Each of the top three priority areas identified in Question 1 of the survey was assigned as a guiding theme for one of the three breakout sessions (Figure 2). The results of the remaining survey questions were presented to the group, and participants were asked to keep these key topics in mind throughout the discussion period (Figure 3). The questions used by moderators to guide each breakout session are presented in Table 2.

Each group simultaneously discussed the same priority topic, and then reconvened as a whole group to share highlights from their discussions. This process was repeated for three rounds, after which all participants had discussed each of the top three priority topics (Figure 1).

\section{Groupthink: Research Questions and Logistics Planning}

Following the three small-group breakout sessions at the end of day one, workshop attendees re-joined as a whole to develop research questions to address the areas highlighted and discussed during the three breakout sessions.
The research questions generated through group discussion were collated by the main rapporteur and a researcher at the end of the first day of the workshop and presented to the workshop participants at the beginning of day two. At this time, group discussion for the development of grant proposals and study logistics planning took place, centered around the research questions.

\section{Results and Discussions Workshop Survey}

In response to the first survey question (Table 1), the top two areas that participants believed must be addressed to improve the health and well-being of mothers and infants affected by HIV were: 1. ART education, safety, and adherence, and 2. an increased understanding of the health risks for infants who are HEU (Figure 2). Safe breastfeeding practices and social support for mothers, including support for disclosure of HIV infection status and treatment non-adherence, were tied for the third most important areas indicated by workshop attendees. Consensus to eliminate social support for mothers as a guiding topic for a breakout session was reached through group discussion, under the premise that this theme would underlie the discussions of all other areas. Thus, the three priority topics set as guiding themes for the three small-group 


\title{
What key concerns must be addressed when aiming to improve the health and well-being of mothers and infants affected by HIV?
}

\author{
ART education, safety and adherence \\ Understanding health risks for \\ infants who are HEU \\ Safe breastfeeding practices
}

Social support for mothers

Adequate antenatal care

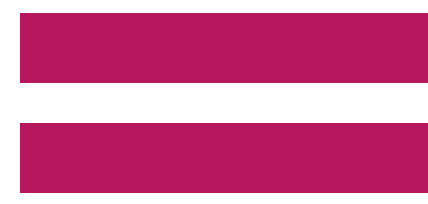

Nutrition
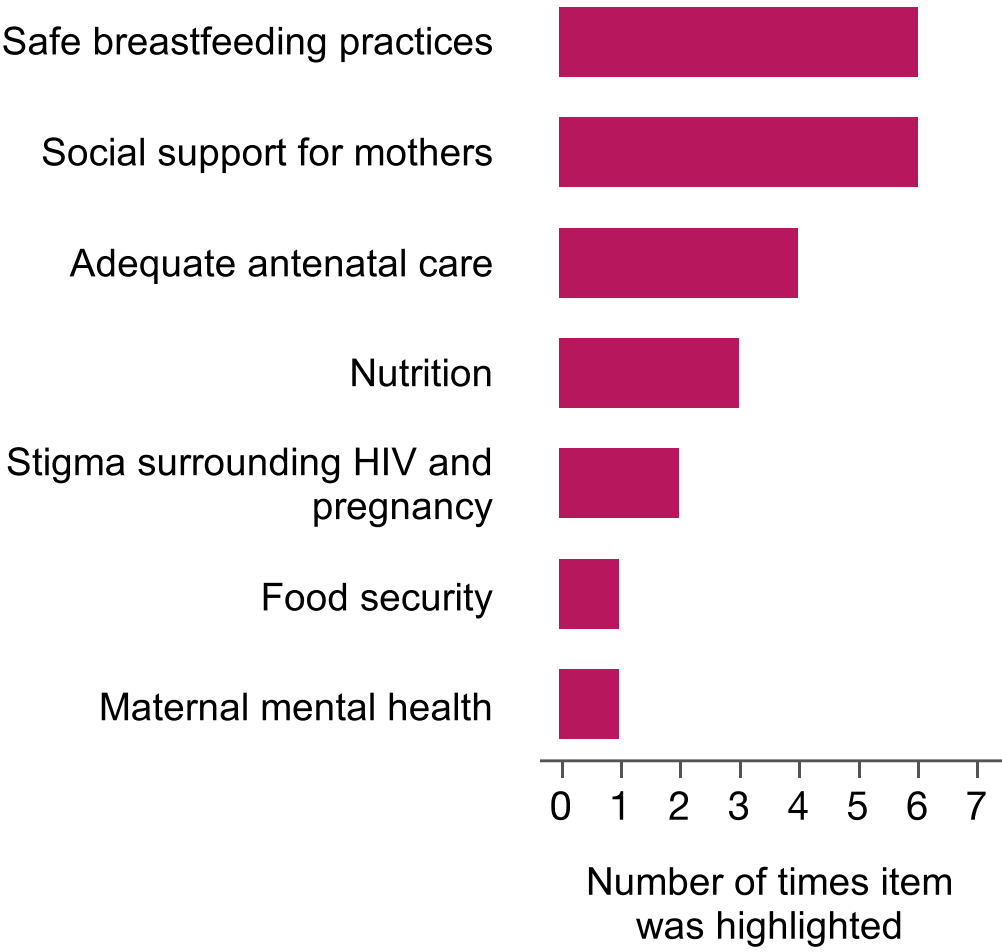

Figure 2 Final results to question I of the workshop surveys following two rounds (via email and in-person) of feedback from workshop participants. ART education, safety, and adherence, understanding health risks for infants who are HEU, and safe breastfeeding practices were identified as the top key areas that must be addressed when aiming to improve health and well-being of mothers and infants affected by HIV, and were chosen as guiding discussion topics for breakout groups.

Abbreviations: ART, antiretroviral therapy; HEU, HIV-exposed, uninfected.

breakout sessions were: 1 . ART education, safety, and adherence, 2. Understanding the health risks for infants who are HEU, and 3. Safe breastfeeding practices for people living with HIV and their infants.

Participants highlighted heterogeneity among cohorts of infants who are HEU, poor ART adherence and treatment complications, and barriers to long term follow-up as the top three significant key challenges that they foresee for the future of maternal-infant HIV research (Figure 3A). Infant growth and development, infant immunological development and function, and maternal physical health were the top three key health outcomes, respectively, that were considered most important to measure when assessing the health trends of infants and mothers affected by
HIV (Figure 3B). Food security and/or quality of food, accessibility to antenatal care and maternal physical health were most frequently ranked in the top three preconception or pregnancy factors that must be evaluated and addressed in order to enhance the well-being and safety of pregnancy in people living with HIV (Figure 3C), while breastfeeding practices, maternal mental health and well-being, and maternal physical health were most frequently ranked as the top postpartum factors that are critical to consider in order to enhance the development of infants and children affected by HIV (Figure 3D).

Participants were asked to brainstorm key components that would be essential to ensure the success of an 
What are 3 significant key challenges you foresee for the future of maternal-infant HIV research?

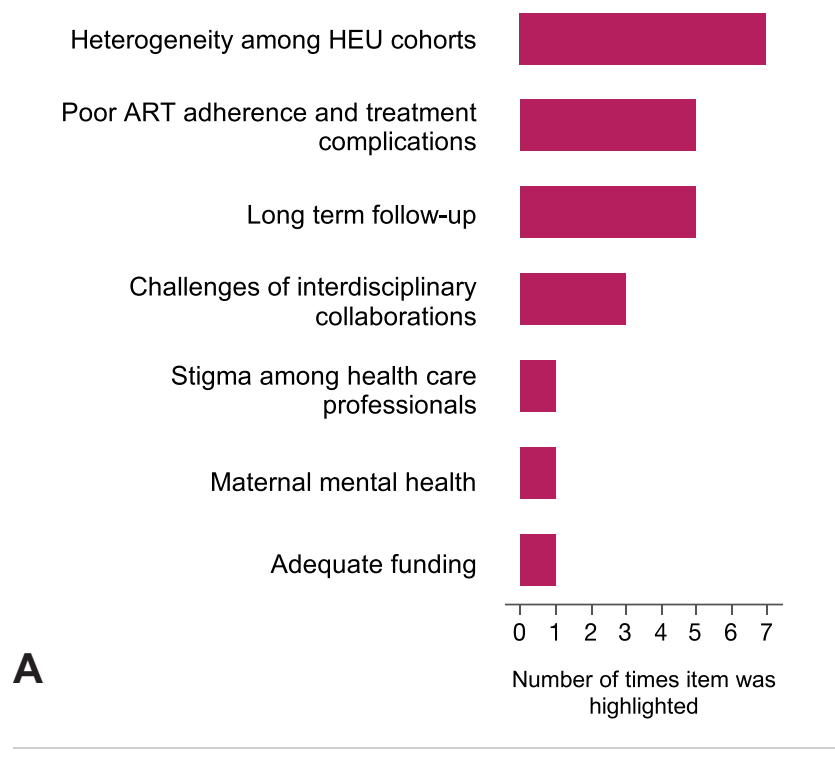

\begin{abstract}
What pre-conception or pregnancy factors must be evaluated and addressed in order to enhance the pregnancy environment in women affected by HIV?
\end{abstract}

Food security and/or quality of food

Accessibility of antenatal care

Maternal physical health

Maternal mental health and wellbeing

Support (partner, family, community)
Maternal level of education attained
Maternal socio-economic characteristics

Home environment

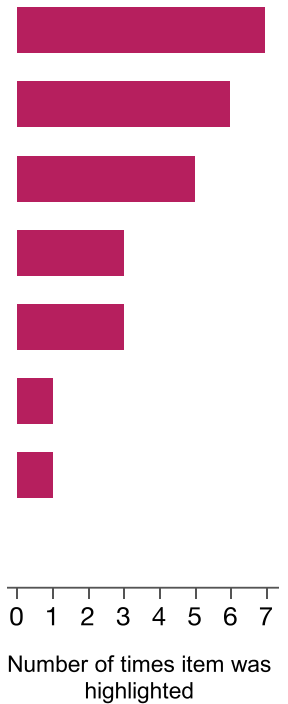

C
What 3 key health outcomes are important to measure when assessing health trends of infants and mothers affected by HIV?

Infant growth and development

Development and function of the infant immune system

Maternal physical health

Quality of life

Maternal mental health

Mother-to-child HIV transmission

Postpartum nutrition

Morbidity

Mortality

B
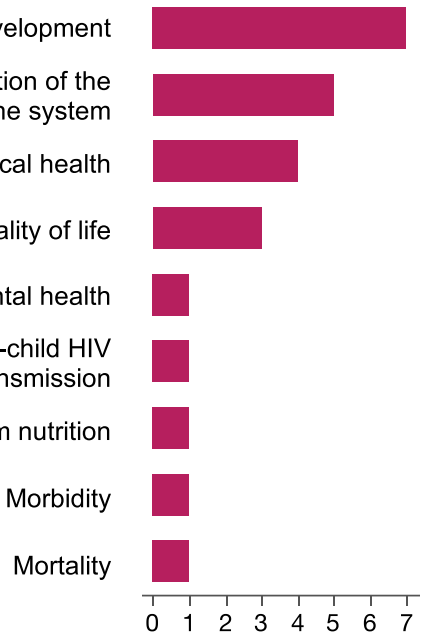

Number of times item was highlighted

What postpartum factors must be evaluated and addressed in order to enhance development of infants and children affected by HIV?

\section{Breastfeeding practices \\ Maternal mental health and well-being \\ Maternal physical health}

Food security and/or quality of food

Support (partner, family, community)

Infant cognitive development

Home environment

Maternal level of education attained

$$
\text { Infant growth }
$$

Accessibility of postnatal care
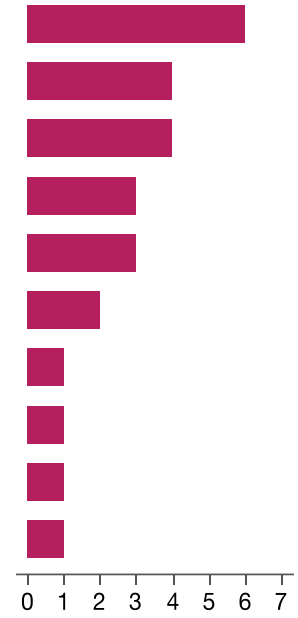

D

Figure 3 Final results to questions 2-3, 5-6 of the workshop surveys following two rounds (via email and in-person) of feedback from workshop participants. Ideas generated in panels (A-D) supported and generated discussions throughout the breakout session and whole group discussions.

Abbreviations: ART, antiretroviral therapy; HEU, HIV-exposed, uninfected.

interdisciplinary research programme aimed at improving the health outcomes of mothers and/or infants affected by HIV in reaching its goals (Figure 4A). The importance of a longitudinal study design, an organized research programme structure, and established mechanisms to update study team members regularly and cohesively was stressed. Participants highlighted that it is critical to set a clear vision that the overall research project and substudies within are trying to achieve. The importance of engaging partners and/or fathers in the research programme was agreed upon, and participants stressed the importance of integrating intervention approaches that target socioeconomic factors and the home environment. Participants agreed that the project must incorporate 
Table 2 Guiding Questions Used by Moderators to Lead SmallGroup Breakout Sessions

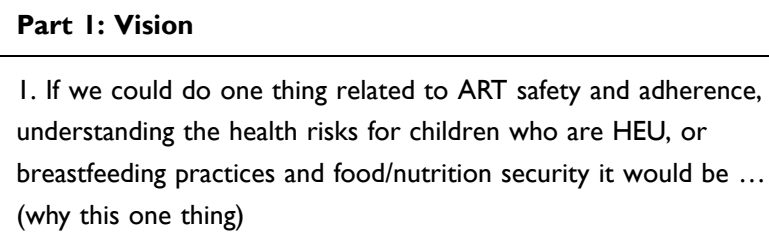

I. If we could do one thing related to ART safety and adherence, understanding the health risks for children who are HEU, or breastfeeding practices and food/nutrition security it would be ... (why this one thing)

2. What are the gaps in the present knowledge related to this one thing?

3. What are the milestones along the way to completing this one thing?

4. What are the stakes or consequences if this does not get done or if it does not go well?

5. What is an example of success?

\section{Part 2: Specifying}

I. What specific research questions are we looking to answer?

2. How will the proposed research questions contribute to knowledge and understanding of the issue (the one thing)?

3. What are our specific aims/objectives?

4. What samples and/or data do we need to answer the research questions?

5. What research design will be most effective in answering the research questions?

6. What components would be most important to ensure the success of this research project in reaching its goals?

Abbreviations: ART, antiretroviral therapy; HEU, HIV-exposed, uninfected.

measurements for all key outcomes (biological and social; infant growth and developmental measures, maternal nutritional status, functional capacity, and mental and physical health states). When asked what strategies for intervention were thought to be essential to prevent or lessen the adverse impact of HIV-exposure for women living with HIV and their infants, education about ART to enhance adherence, early interventions to mitigate the adverse effects of HEU, and ensuring antenatal care access were highlighted most frequently (Figure 4B).

\section{Breakout Session Discussion Highlights ART Education, Safety, and Adherence}

Priority topics concerning ART education, safety and adherence were: misinformation related to treatment expectations and outcomes; education and health literacy for women and their support systems; and the inaccessibility of appropriate counselling to support women with their treatment adherence. The Siyakhula study participants who attended the workshop shared that misinformation related to the risk, and likelihood, of experiencing side effects associated with ART directly relates to treatment non-adherence (Figure 5). They also felt that there was widespread misinformation about the health risks for women living with HIV and their children in the event of inadequate treatment adherence, namely during pregnancy and breastfeeding. Misconceptions about the quality of life that is attainable for those living with HIV, that is, misunderstandings of the true benefits to be gained from treatment regimens, also arose as a potential contributor to disengagement from care. Workshop discussions stressed the importance of addressing different types of non-adherence, such as poor or partial adherence versus total non-adherence, with tailored approaches.

Increasing the health literacy of women living with HIV should be a priority, in order to equip women with information about their own health, provide them with the tools they need to engage in their care, and advocate for what they need. Women's support systems are key, and education efforts aimed at these individuals, namely partners, fathers of their children, families, close friends, and community health workers are currently inadequate, and have the potential to improve their ability to support women with their treatment adherence.

Importantly, the intersection of ART adherence and safe breastfeeding practices was discussed in depth, and participants expressed that the importance of treatment adherence while breastfeeding must be effectively communicated to mothers. Health literacy related to HIV viral load is important to ensure women are equipped with the knowledge to understand and discuss the management of their viral load results and appreciate the implications of these results for safe breastfeeding practices.

It was stressed that spaces for women to honestly, and safely, communicate feelings of depression, disengagement with care, and difficulties surrounding treatment management are critical but not always readily available. This may take the form of peer support groups, or group or one-on-one counselling. Interventions to increase treatment adherence must also be aimed at health workers providing care. Perspectives from those with lived experience need to be sought, particularly to understand why women may stop taking ART. Health workers must become more familiar with specific contributors to nonadherence in order to address them. Patient-centred efforts 
A What components are essential to ensure the success of an interdisciplinary research programme aimed at improving the health outcomes of mothers and/or infants affected by HIV in reaching its goals

Longitudinal study design

Organized research programme structure

Mechanisms to regularly and cohesively update the study team

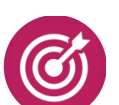

A clear aim for the overall research project

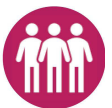

Engagement of partners and/or fathers

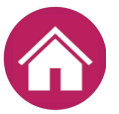

Integration of interventions that target socioeconomic factors and the home environment

\title{
B
}

\section{What strategies for intervention are essential to prevent or lessen the adverse impacts of HIV-exposure for women living with HIV, and their infants?}

\author{
Education about ART to enhance adherence \\ Early interventions to mitigate adverse effects of HEU \\ Ensure antenatal care access \\ Improve food security \\ Increase understanding of HEU phenotype \\ Public education about HIV and treatment \\ Comprehensive health education

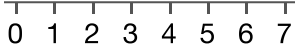 \\ Number of times item was \\ highlighted
}

Figure 4 Final results to questions $4(\mathbf{A})$ and 7 (B) of the workshop surveys following two rounds (via email and in-person) of feedback from workshop participants. Abbreviations: ART, antiretroviral therapy; HEU, HIV-exposed, uninfected.

will assist women to communicate the support they need to help them adhere to their medication.

\section{Understanding the Health Risks for Infants Who are HEU}

The ongoing risk of transmission, low breastfeeding rates, and a poor understanding of the HEU phenotype were highlighted as key contributors to health risks for children who are HEU. As vertical transmission remains a health risk, participants expressed a need for more active polymerase chain reaction (PCR) testing for children who are HIV-exposed, particularly throughout the breastfeeding period. At the time of this workshop, South African guidelines for the prevention of mother-to-child transmission of HIV recommended that PCR testing be performed at birth and 10 weeks, and again at 18 weeks for infants who received 12 weeks of nevirapine prophylaxis. ${ }^{19}$ Rapid HIV antibody testing is recommended at 18 months for all children who are HIV-exposed. ${ }^{19}$ Participants also expressed a need for more frequent maternal viral load testing throughout the breastfeeding period to 


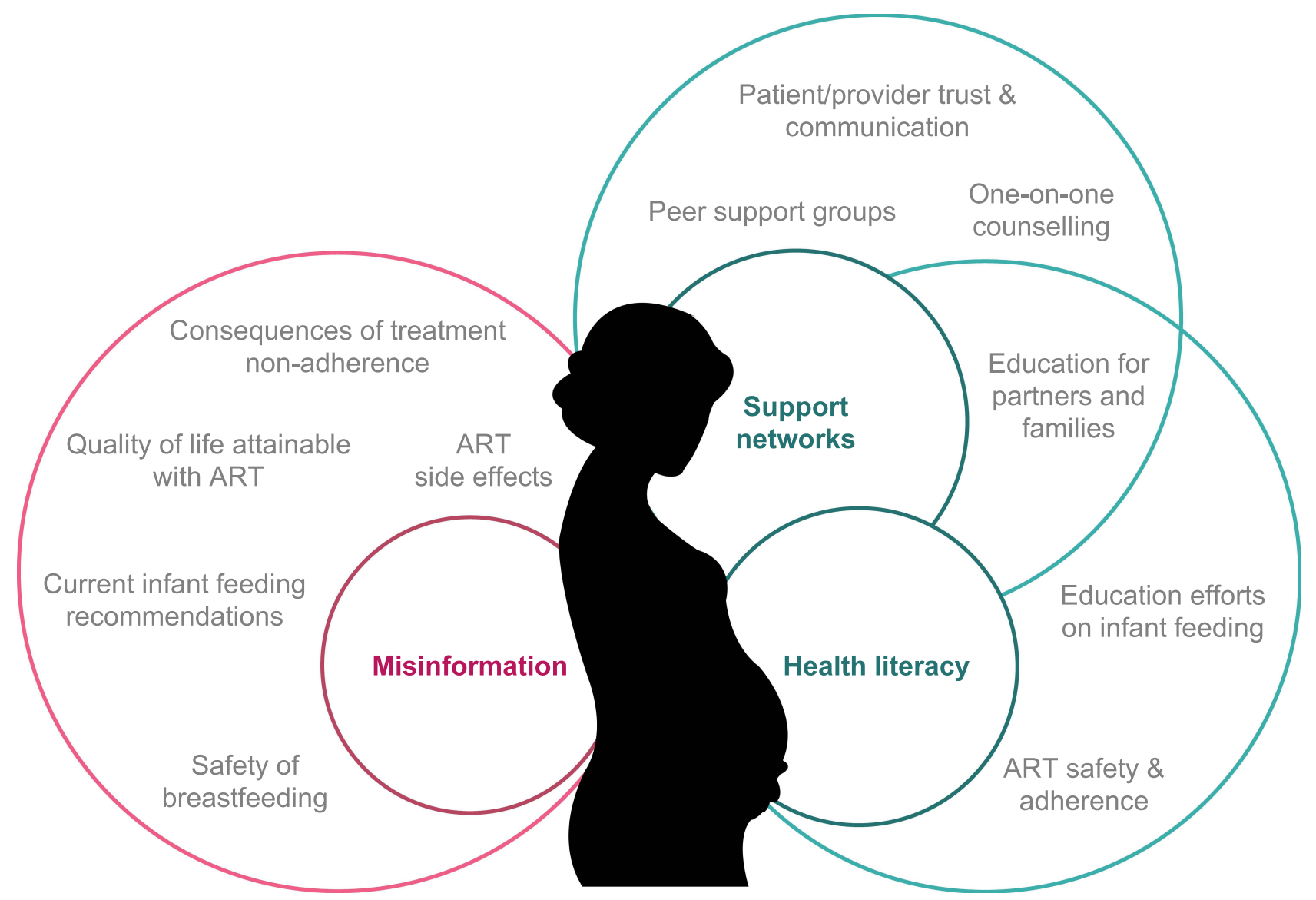

Barriers

\section{Opportunities}

Figure 5 Summary of perspectives shared by the women with lived experience at the workshop. Illustration of the barriers and opportunities to improve the health and well-being of women and children affected by HIV highlighted by the study participants in attendance throughout breakout group and whole-group discussions.

ensure that accurate and relevant counselling regarding safe breastfeeding practices is being provided to women. The low rates of breastfeeding among people living with HIV and the absence of breastmilk as a nutrition source for infants who are HEU may heighten their risk for common infections and compromise infant immunological development.

Short, medium, and long-term neurodevelopmental, immunological and growth outcomes in children who are HEU remain poorly described. Modifiable factors that contribute to these outcomes, including the role of breastfeeding practices, food security and maternal nutrition are not well evaluated, suggesting that there are influential variables yet to be examined. The long-term health risks associated with in utero ART exposure are also not well understood. The high heterogeneity among infants who are HEU requires community-based, individualized approaches to understand and address different health profiles and challenges.

\section{Safe Breastfeeding Practices}

Misinformation related to the safety of breastfeeding, the accessibility of current recommendations, and a limited understanding of the composition of the breastmilk of people living with HIV emerged as common concerns. First, the study participants in attendance highlighted mixed messaging related to guidance for breastfeeding, formula, and mixed feeding as a barrier to meeting the recommendations for exclusive breastfeeding (EBF) and, in fact, any breastfeeding. The discussions centered around the idea that after over a decade of recommending that women living with HIV in South Africa should not breastfeed, the normality around breastfeeding had shifted, and persisting societal stigma surrounding the safety of breastfeeding while living with HIV presents barriers for new mothers. Clear information related to all feeding options, including how to safely breastfeed at different periods of 
infant development, needs to be better communicated to mothers so that they are equipped with the information needed to make informed infant-feeding decisions. Education efforts to support breastfeeding need to be extended to the support systems of women, and the benefits of breastfeeding for both mothers and babies need to be better communicated to individuals engaged in their care. Participants expressed that efforts to breastfeed are challenging for women when they are not supported by those around them, given that the mother may not be the sole caretaker of the child.

Participants also agreed that policies to support breastfeeding need to be community-centred and led and must also consider ongoing feeding practices for young children beyond the period of breastfeeding. Current recommendations related to the time period of breastfeeding ( 6 months EBF, followed by continued breastfeeding for up to 24 months ${ }^{20}$ with the addition of complementary feeds) are often unattainable. Requirements to return to work, social pressures that doubt the safety of breastfeeding with HIV infection, and the high energy demands of breastfeeding were all cited as barriers. Clear recommendations need to be made available to women for whom the current guidelines are not accessible, allowing them to seek breastfeeding guidance that meets their needs. It was expressed by health workers that there is misinformation about the benefits of any breastfeeding versus EBF, leading mothers for whom EBF is not accessible to opt for no breastfeeding at all. Ongoing formula feeding also presents a socioeconomic burden for families. Support for mothers to work while breastfeeding is critical and may require advocacy for normalization of breastfeeding in the workplace, and increased education related to expressing and storing breastmilk. The energy demands that breastfeeding requires may be a key obstacle in situations where maternal malnutrition is a coexisting challenge.

Group discussions also highlighted that current data on how HIV infection and ART may influence breastmilk composition, and to what extent, remain limited. It has been reported that levels of fucosylated human milk oligosaccharides in breast milk are inversely associated with infant mortality among infants who are HEU throughout the breastfeeding period, ${ }^{21}$ and there may be subtle, but potentially biologically important, differences in the immunoglobulin profiles of breastmilk from people living with versus without HIV. ${ }^{22,23}$ An improved understanding of the breastmilk profiles of people living with HIV is critical given the promotion of breastfeeding for mothers living with HIV.

\section{Groupthink: Research Questions}

Research questions developed by the group to address ART education, safety, and adherence, understanding the health risks for children who are HEU, and safe breastfeeding practices are presented in Table 3. Priority research questions on breastfeeding practices and food and nutrition security, as well as understanding the health risks for children who are HEU, have since been integrated by group members into the Siyakhula study, to better understand the perinatal environmental factors that shape development and health in infants who are HEU. Group members have also built on key discussions to evaluate the evidence about how early nutritional targets may be optimised to improve neurodevelopmental outcomes for infants who are HEU. ${ }^{24}$

\section{Workshop Feedback}

Feedback from workshop participants overall was positive. Representatives from health programmes expressed

Table 3 Research Questions Developed Through Small-Group Breakout Session Discussions That Aim to Address Key Concerns Highlighted as Guiding Themes

\begin{tabular}{l} 
ART education, safety, and adherence \\
\hline $\begin{array}{l}\text { I. What are the drivers of non-adherence? Is it true non-adherence, } \\
\text { or are they seeking health care elsewhere? }\end{array}$ \\
\hline $\begin{array}{l}\text { 2. What can we do to promote adherence? How can we increase } \\
\text { disclosure of non-adherence? }\end{array}$ \\
\hline Understanding the health risks for children who are HEU \\
\hline $\begin{array}{l}\text { I. What components of the HEU phenotype are associated ART } \\
\text { exposure (mom vs infant)? }\end{array}$ \\
\hline $\begin{array}{l}\text { 2. How does ongoing HIV viral replication alter the HEU phenotype? } \\
\text { Will switching to new therapies effect the HEU phenotype? }\end{array}$ \\
\hline 3. What is the weight of the various risk factors that are contributing? \\
\hline 4. When do different types of neurodevelopmental dysfunctions emerge? \\
\hline Breastfeeding practices and food/nutrition security \\
\hline $\begin{array}{l}\text { I. Is there a difference in infant outcomes associated with mixed vs } \\
\text { exclusive breastfeeding? }\end{array}$ \\
\hline 2. Does food security impact BF practices? \\
\hline 3. How does nutrition security affect BM composition and infant growth? \\
\hline $\begin{array}{l}\text { 4. How can we increase length of BF? What prevents mothers from } \\
\text { meeting the guidelines? }\end{array}$ \\
\hline
\end{tabular}

Abbreviations: ART, antiretroviral therapy; HEU, HIV-exposed, uninfected; BF, Breastfeeding; BM, Breastmilk. 
that the data shared by academics were informative, and highlighted engagement between clinicians and academics as an important means for translating the latest research findings into care. Representatives from all professions valued the perspectives shared by the study participants in attendance and agreed that their viewpoints were invaluable. The workshop helped to highlight the interdisciplinary roles that are required to make a large clinical cohort study run by a multidisciplinary team successful, and an increased sense of place and engagement within the project was voiced among professionals from all areas.

\section{Summary and Conclusions}

A visual summary of the workshop's discussion highlights (adapted from an infographic generated following the workshop $^{25}$ ) is presented in Figure 6. Misinformation between people living with HIV and health workers is a key barrier to ART adherence and EBF, which may exacerbate the health risks faced by children who are HEU. Societal stigma related to

\section{Priority areas to address to improve the health and well-being of mothers and infants affected by HIV}
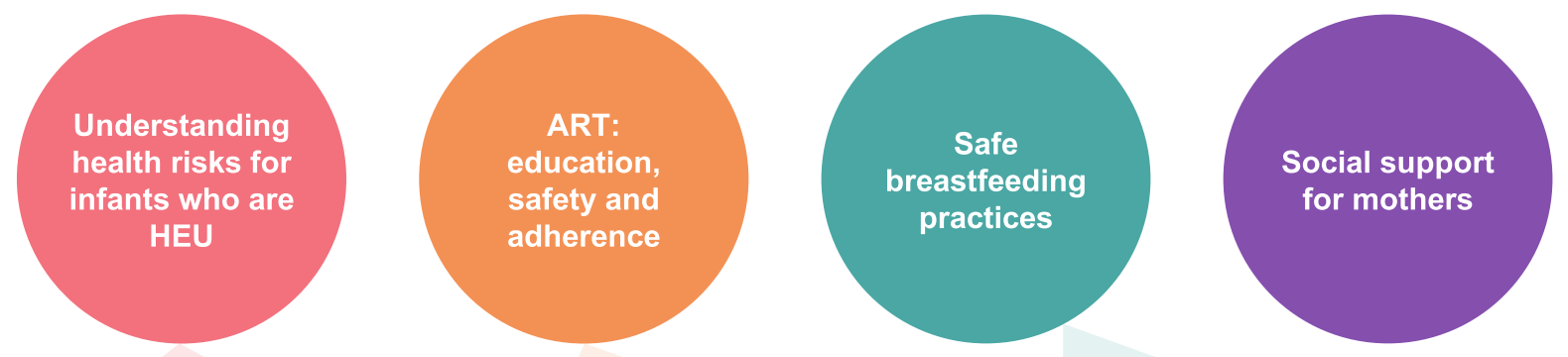

Summary of multi-stakeholder discussion highlights

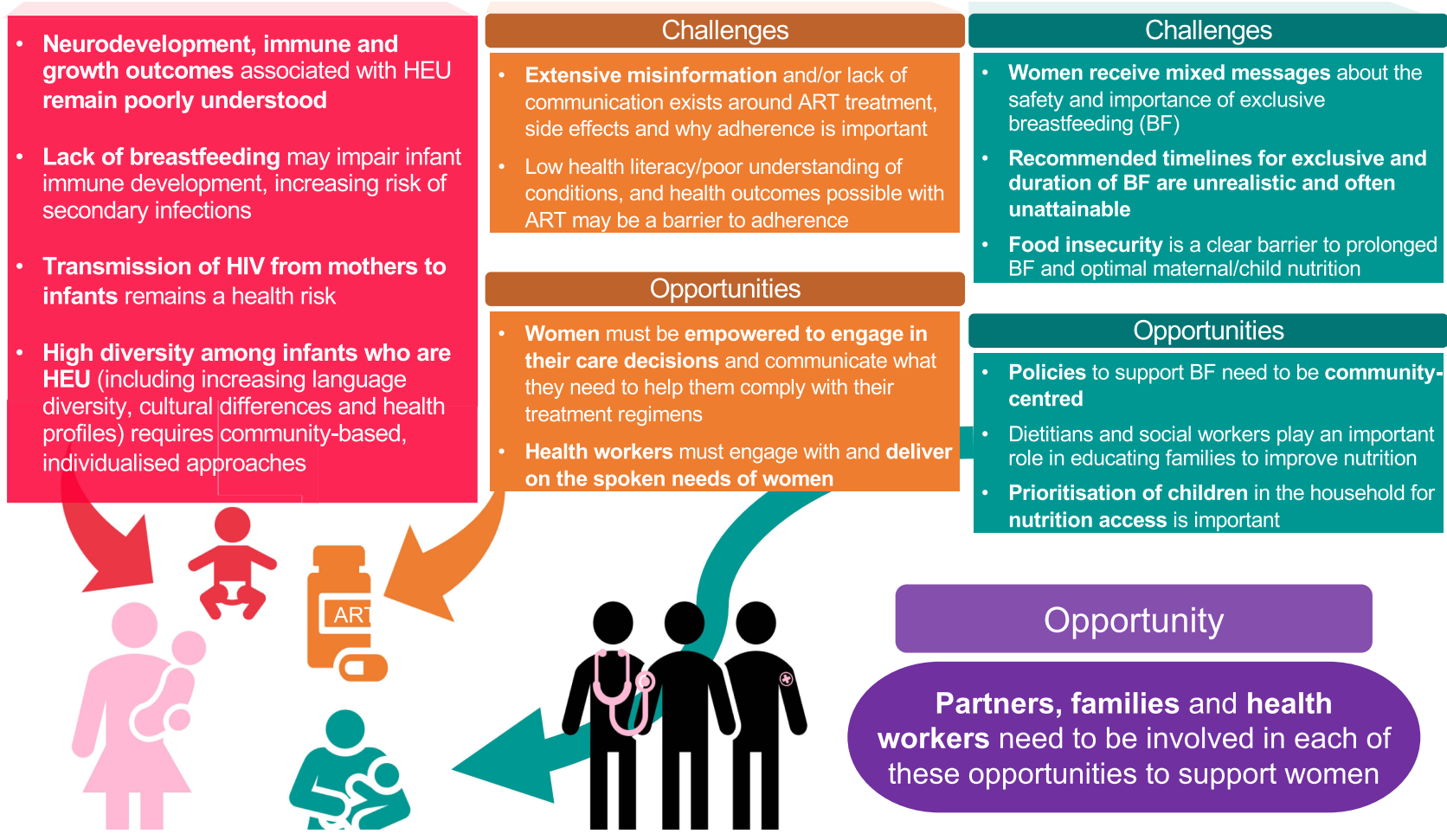

Figure 6 Summary of priority areas to address, and key challenges and opportunities identified through workshop discussions. To view the full infographic, visit: http://doi. org/ $10.528 \mathrm{I} /$ zenodo.3479460.

Abbreviations: ART, antiretroviral therapy; HEU, HIV-exposed, uninfected; BF, Breastfeeding. 
HIV is a barrier to disclosure of HIV status and supportseeking for treatment adherence and contributes to apprehension towards breastfeeding. The support systems that surround women are key, and these people and groups need to be educated about how to support women with regards to ART adherence and breastfeeding. Intervention efforts to improve ART adherence, promote breastfeeding and reduce health risks for children who are HEU need to be community-centred given the high heterogeneity among HEU infants, and diversity in barriers to treatment adherence and breastfeeding. Pretransmission education should be a priority to reduce new HIV infections and equip individuals who do become infected with the information needed to make decisions about their care. Research questions must further investigate the effects of cooccurring exposure to HIV and ART in the womb, and malnutrition on neurodevelopment, immunological and growth outcomes in children who are HEU. By bringing together multidisciplinary stakeholders, we can close knowledge gaps pertaining to HIV infection in the context of maternal and child health through the co-development of research questions and interventions that are salient to affected communities, health care providers and policy makers alike, all to ensure better health across generations.

\section{Acknowledgments}

The authors would like to thank all participants and presenters for their attendance and contributions to the workshop, as well as Cathy Bezuidenhout, Clara Nkadimeng, Danie Thirion, and the rest of the staff at the University of Pretoria's Research Centre for Maternal, Fetal, Newborn \& Child Health Care Strategies for their support in organizing the workshop. Thank you also to the workshop rapporteurs, Louise du Toit, Andrea Prinsloo, Mieke van der Mescht and Marina White, for capturing the workshop proceedings.

\section{Author Contributions}

All authors contributed to data analysis, drafting or revising the article, have agreed on the journal to which the article will be submitted, gave final approval of the version to be published, and agree to be accountable for all aspects of the work. Conceptualization/design: MW, KC, UT, TR; Data acquisition and analysis/interpretation: MW, LDT, KC; writing/revising and final approval: MW, KC, UT, TR, LDT.

\section{Funding}

This workshop was funded by the Canadian Institutes for Health Research and the Collaborative Initiative for Paediatric HIV Education and Research.

\section{Disclosure}

Prof. Dr. Theresa Rossouw report Speaker fees received from Merck - not related to the work presented in this publication. The authors reports no other conflicts of interest in this work.

\section{References}

1. UNAIDS. Fact Sheet - World AIDS Day. Global HIV statistics; 2020.

2. Moodley T, Moodley D, Sebitloane M, Maharaj N, Sartorius B. Improved pregnancy outcomes with increasing antiretroviral coverage in South Africa. BMC Pregnancy Childbirth. 2016;16(1):35. doi:10.1186/s12884-016-0821-3

3. UNAIDS, AIDSinfo. Elimination of mother-to-child transmission: HIV-exposed children who are uninfected. Available from: https:// aidsinfo.unaids.org/. Accessed July 12, 2021.

4. Slogrove AL, Powis KM, Johnson LF, Stover J, Mahy M. Estimates of the global population of children who are HIV-exposed and uninfected, 2000-18: a modelling study. Lancet Glob Health. 2020;8(1): e67-e75. doi:10.1016/S2214-109X(19)30448-6

5. Springer PE, Slogrove AL, Laughton B, et al. Neurodevelopmental outcome of HIV-exposed but uninfected infants in the Mother and Infants Health Study, Cape Town, South Africa. Trop Med Int Health. 2018;23(1):69-78. doi:10.1111/tmi.13006

6. da Silva KM, de Sá C, Carvalho R. Evaluation of motor and cognitive development among infants exposed to HIV. Early Hum Dev. 2017;105:7-10. doi:10.1016/j.earlhumdev.2016.12.013

7. White M, Feucht UD, Duffley E, et al. Does in utero HIV exposure and the early nutritional environment influence infant development and immune outcomes? Findings from a pilot study in Pretoria, South Africa. Pilot Feasibility Stud. 2020;6(1):192. doi:10.1186/s40814020-00725-8

8. Omonaiye O, Kusljic S, Nicholson P, Manias E. Medication adherence in pregnant women with human immunodeficiency virus receiving antiretroviral therapy in Sub-Saharan Africa: a systematic review. BMC Public Health. 2018;18(1):805. doi:10.1186/s12889-018-5651-y

9. WHO. Consultative meeting on nutrition interventions for improving the prevention, care and management of HIV/AIDS. 2003. Available from: https://www.who.int/nutrition/publications/meet_nut_interven tions_hivaids.pdf?ua=1. Accessed July 12, 2021.

10. Anema A, Vogenthaler N, Frongillo EA, Kadiyala S, Weiser SD. Food insecurity and HIV/AIDS: current knowledge, gaps, and research priorities. Curr HIV/AIDS Rep. 2009;6(4):224-231. doi:10.1007/s11904-009-0030-z

11. Dadabhai S, Gadama L, Chamanga R, et al. Pregnancy outcomes in the era of universal antiretroviral treatment in Sub-Saharan Africa (POISE Study). J Acquir Immune Defic Syndr. 2019;80(1):7-14. doi:10.1097/QAI.0000000000001875

12. Wadhwa PD, Buss C, Entringer S, Swanson JM. Developmental origins of health and disease: brief history of the approach and current focus on epigenetic mechanisms. Semin Reprod Med. 2009;27(5):358-368. doi:10.1055/s-0029-1237424

13. Domecq JP, Prutsky G, Elraiyah T, et al. Patient engagement in research: a systematic review. BMC Health Serv Res. 2014;14:89. doi:10.1186/1472-6963-14-89

14. Fink A, Kosecoff J, Chassin M, Brook RH. Consensus methods: characteristics and guidelines for use. Am J Public Health. 1984;74 (9):979-983. doi:10.2105/AJPH.74.9.979

15. Hasson F, Keeney S, McKenna H. Research guidelines for the Delphi survey technique. $J$ Adv Nurs. 2000;32(4):1008-1015. doi:10.1046/ j.1365-2648.2000.t01-1-01567.x

16. Delbecq AL, Van De Ven AH. Model for problem identification and program planning. J Appl Behav Sci. 1971;7(4):466-492. doi: $10.1177 / 002188637100700404$ 
17. Dalkey NC. The Delphi Method: An Experimental Study of Group Opinion. Rand Corporation. 1969.

18. Van de Ven AH, Delbecq AL. The nominal group as a research instrument for exploratory health studies. Am J Public Health. 1972;62(3):337-342. doi:10.2105/ajph.62.3.337

19. National Department of Health South Africa. National Consolidated Guidelines: For the Prevention of Mother-To-Child Transmission of HIV (PMTCT) and the Management of HIV in Children, Adolescents and Adults. 2015.

20. WHO. Infant Feeding for the Prevention of Mother-To-Child Transmission of HIV. WHO; 2019.

21. Kuhn L, Kim H-Y, Hsiao L, et al. Oligosaccharide composition of breast milk influences survival of uninfected children born to HIV-infected mothers in Lusaka, Zambia. $J$ Nutr. 2015;145 (1):66-72. doi:10.3945/jn.114.199794
22. Shapiro RL, Lockman S, Kim S, et al. Infant morbidity, mortality, and breast milk immunologic profiles among breast-feeding HIV-infected and HIV-uninfected women in Botswana. J Infect Dis. 2007;196(4):562-569. doi:10.1086/519847

23. Pedersen SH, Wilkinson AL, Andreasen A, et al. Longitudinal analysis of mature breastmilk and serum immune composition among mixed HIV-status mothers and their infants. Clin Nutr. 2016;35 (4):871-879. doi:10.1016/j.clnu.2015.05.016

24. White M, Connor KL. In utero HIV exposure and the early nutritional environment influence infant neurodevelopment: findings from an evidenced review and meta-analysis. Nutrients. 2020;12(11):3375. doi:10.3390/nu12113375

25. White M, Connor KL. Siyakhula collaborative workshop: infographic. 2019. doi:10.5281/zenodo.3479460
Journal of Multidisciplinary Healthcare

\section{Publish your work in this journal}

The Journal of Multidisciplinary Healthcare is an international, peerreviewed open-access journal that aims to represent and publish research in healthcare areas delivered by practitioners of different disciplines. This includes studies and reviews conducted by multidisciplinary teams as well as research which evaluates the results or conduct of such teams or healthcare processes in general. The journal

\section{Dovepress}

covers a very wide range of areas and welcomes submissions from practitioners at all levels, from all over the world. The manuscript management system is completely online and includes a very quick and fair peer-review system. Visit http://www.dovepress.com/testimonials. php to read real quotes from published authors. 